\title{
Blutglucose und Seruminsulin nach oraler Applikation von Glucose und Stärkesirup in unterschiedlicher Dosierung
}

\author{
H. Förster, M. Haslbeck, C.-A. Geser und H. Mehnert
}

Institut für vegetative Physiologie der Universität Frankfurt (Direktor: Prof. Dr. E. Heinz)

Forschergruppe Diabetes (Gemeinsame Leitung: Prof. Dr. H. Mehnert und Prof. Dr. O. Wieland) und 3. Medizinische Abteilung des Städtischen Krankenhauses München-Schwabing (Chefarzt: Prof. Dr. H. Mehnert), Deutschland

Eingegangen am 23. Juli 1969

\begin{abstract}
Blood glucose and serum insulin after oral loading with glucose and starch syrup in varying doses

Summary. 6 groups consisting of $8-14$ healthy control persons received $50 \mathrm{~g}, 100 \mathrm{~g}$ or $200 \mathrm{~g}$ of glucose or of a starch-hydrolyzate preparation by mouth. Blood sugar changes were largely independent of the type or amount of carbohydrate given. Serum insulin values, however, showed a definite correlation with the amount of ingested carbohydrate. After $50 \mathrm{~g}$ of carbohydrate the rise of sorum insulin was about $50 \mu \mathrm{U} / \mathrm{ml}$; after $100 \mathrm{~g}$ of carbohydrate it reached $90-100 \mu \mathrm{U} / \mathrm{ml}$. There was no further rise of maximal values after ingestion of larger amounts of carbohydrate, but the normalization of insulin levels was markedly delayed. From the pattern of serum insulin levels after ingestion of large amounts of carbohydrates, it can be concluded that glucose absorption is not finish ed at $120 \mathrm{~min}$ despite normal blood glucose values. As indicated by these results, $100 \mathrm{~g}$ of glucose or starch-syrup is better suited for diagnostic purposes than is $50 \mathrm{~g}$ of carbohydrates. Only the ingestion of $100 \mathrm{~g}$ of carbohydrates stimulates insulin release sufficiently, which is of special diagnostic interest for the most significant interval of the test, i.e. $2 \mathrm{~h}$ after ingestion of the carbo. hydrate load.
\end{abstract}

Glucose sanguin et insuline sérique après charge orale de glucose et de sirop d'amidon à différentes doses

Résumé. 6 groupes de $8-14$ sujets en bonne santé ont reçu $50 \mathrm{~g}, 100 \mathrm{~g}$ ou $200 \mathrm{~g}$ de glucose ou d'une préparation d'hydrolysat d'amidon par voie orale. Les modifications de la glycémie étaient largement indépendantes du type ou de la quantité d'hydrates de carbone administrés. Les valeurs de l'insuline sérique, par contre, montraient une corrélation nette avec la quantité d'hydrates de carbone ingérés. Après $50 \mathrm{~g}$ d'hydrates de carbone, l'augmentation de l'insuline sérique était environ de $50 \mu \mathrm{U} / \mathrm{ml}$; après $100 \mathrm{~g}$ d'hydrates de carbone, elle atteignait $90-100 \mu \mathrm{U} / \mathrm{ml}$. Il n'y avait pas d'autres augmentations des valeurs maximales après ingestion de quantités plus grandes d'hydrates de carbone, mais la normalisation des taux d'insuline était nettement retardée. A partir du comporte- ment des taux d'insuline sérique après ingestion de grandes quantités d'hydrates de carbone, on peut conclure que la résorption du glucose n'est pas terminée à $120 \mathrm{~min}$ malgré des valeurs normales de glucose sanguin. Comme l'indiquent ces résultats, $100 \mathrm{~g}$ de glucose ou de sirop d'amidon conviennent mieux à des buts diagnostiques que $50 \mathrm{~g}$ d'hydrates de carbone. Seulement l'ingestion de $100 \mathrm{~g}$ d'hydrates de carbone stimule suffisamment la sécrétion d'insuline, ce qui est d'un intérêt diagnostique spécial pour le moment le plus important du test, c'est-à-dire $2 \mathrm{~h}$ après l'ingestion d'une charge d'hydrates de carbone.

Zusammenfassung. Bei 6 Gruppen stoffwechselgesunder Personen (8-14 Probanden pro Gruppe) wurden orale Belastungen mit $50 \mathrm{~g}, 100 \mathrm{~g}$ oder $200 \mathrm{~g}$ Glucose bzw. Stärkehydrolysat durchgeführt. Die Blutglucoseveränderungen waren von der Art und der Menge der verabreichten Kohlenhydrate weitgehend unabhängig. Bei den Seruminsulinwerten war dagegen eine deutliche Beziehung zur verabreichten Kohlenhydratmenge vorhanden. Nach $50 \mathrm{~g}$ Kohlenhydraten betrug dor Anstieg des Seruminsulins etwa $50 \mu \mathrm{E} / \mathrm{ml}$, nach $100 \mathrm{~g}$ Kohlenhydraten etwa $90-$ $100 \mu \mathrm{E} / \mathrm{ml}$. Eine weitere Erhöhung der verabreichten Kohlenhydratmenge bewirkt zwar keine weitere Steigerung der Maximalwerte, jedoch war die Normalisierung der Seruminsulinwerte stark verzögert. Aus dem Verhalten der Seruminsulinwerte nach Verabreichung großer Kohlenhydratmengen kann geschlossen werden, daß trotz Normalisierung der Blutglucosewerte die Glucoseresorption nach 120 min noch nicht abgeschlossen ist. $100 \mathrm{~g}$ Glucose oder Stärkesirup sind nach diesen Ergebnissen für die orale Belastungsporobe zu diagnostischen Zwecken besser geeignet als $50 \mathrm{~g}$ Kohlenhydrate. Erst bei Einnahme von $100 \mathrm{~g}$ Kohlenhydraten wird die Insulinsekretion ausreichend stimuliert, was besonders für den wichtigsten Zeitpunkt der Untersuchung - zwei Stunden nach Einnahme der Kohlenhydrate - von wesentlicher diagnostiseher Bedeutung ist.

Key-words: oral glucose tolerance test, serum insulin, insulin release, glucose absorption, starch hydrolyzate, diabetes diagnostic.
Die Wechselbeziehung zwischen Blutglucose und Seruminsulin ist seit langem bekannt. In dem Regelkreis ist die Blutglucosekonzentration die Regelgröße. Über die Insulinsekretion des Pankreas wird durch die Blutglucosekonzentration die als Stellgröße fungierende Seruminsulinkonzentration beeinflußt. In den letzten Jahren wurde festgestellt, da $\beta$ auch viele andere Substanzen neben der Glucose die Insulinsekretion des Pankreas stimulieren [6]. Dennoch wurde mehrfach der Versuch gemacht, eine Beziehung zwischen Blutglucosekonzentration und Seruminsulinkonzentration bei Glucosebelastungen zu finden [13, 11, 15]. Eine solche Beziehung ist jedoch nur sinnvoll, wenn außer Glucose keine wesentlichen anderen Faktoren für die Insulinsekretion von Bedeutung wären.

Wir hatten bereits früher vermutet, daß bei oralen Glucosebelastungen die Blutglucosekonzentration schon normalisiert sein kann, obwohl die Glucoseresorption noch nicht abgeschlossen ist [4]. Zur Beant- 
wortung dieser Frage können gleichzeitige Untersuchungen der Blutglucose und des Seruminsulins nach oraler Glucosebelastung dienen. Dabei kann auch zu der für die Diagnostik wichtigen Frage Stellung genommen werden, welche Glucosemenge bei oralen Provokationstests unter besonderer Berücksichtigung des Zweistundenwertes als optimal anzusehen ist. Aus diesen Gründen sollten bei nichtdiabetischen Versuchspersonen orale Belastungen mit unterschiedlichen Glucosemengen durchgeführt werden. Außerdem wurden Untersuchungen mit Stärkesirup durchgeführt, der in anderen Ländern bereits häufig für die Diagnostik verwendet wird.

\section{Methodik}

Bei gesunden normalgewichtigen Versuchspersonen (20-25 Jahre alt) wurden die Belastungen in verschiedener Dosierung durchgeführt. Die Blutglucose wurde enzymatisch mit Glucoseoxydase-Peroxydase
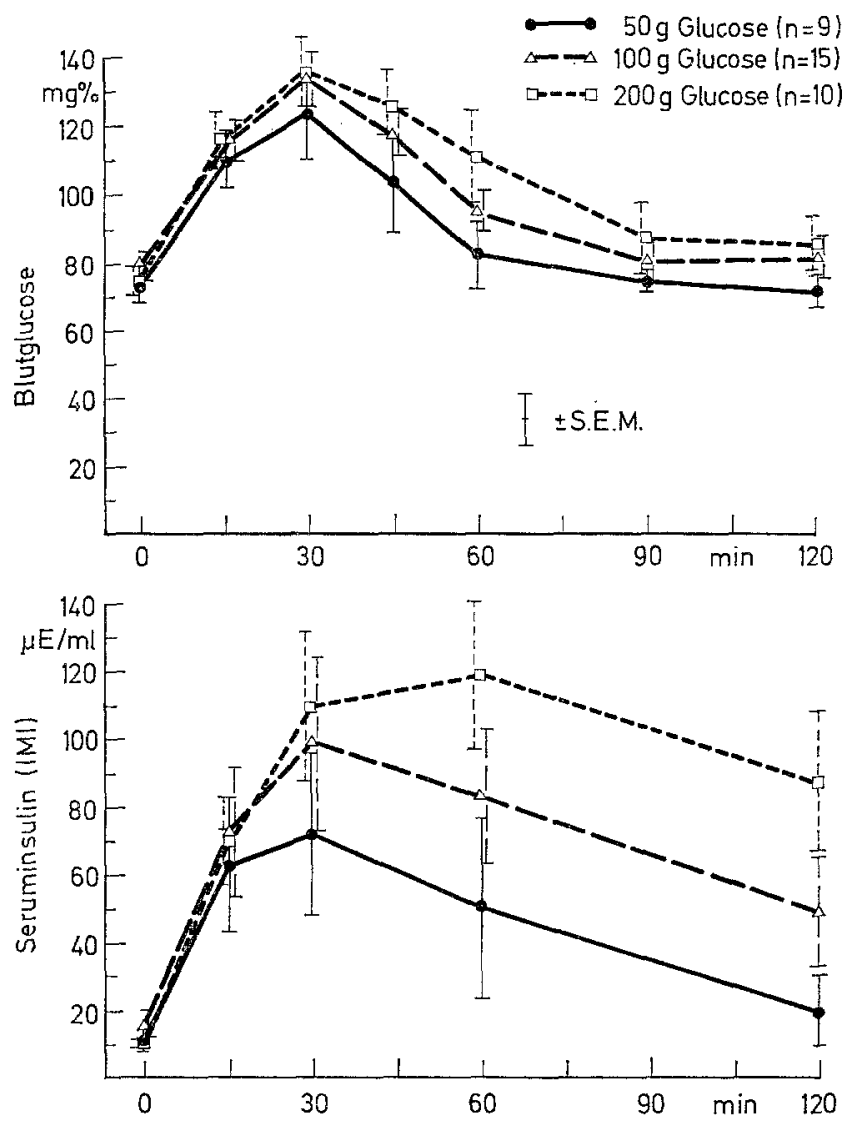

Abb. 1. Verhalten der Blutglucose und des Seruminsulins (IMI) bei jungen gesunden Versuchspersonen nach oraler Verabreichung von Glucose

bestimmt [8], das Seruminsulin wurde immunologisch. nach der Methode von Hales und Randle nachgewiesen [7]. Die Bestimmungen wurden mit venösem Blut durchgeführt. Alle Einzelheiten der Versuchsanord- nung und die Zahl der Probanden sind den Abb. 1 und 2 zu entnehmen.

\section{Ergebnisse}

Die Änderungen der Blutglucose sind weitgehend unabhängig von der verabreichten Glucosemenge (siehe Abb. 1). Lediglich bei einer Dosis von $200 \mathrm{~g}$ Glucose erfolgt die Normalisierung der Blutglucosewerte etwas verzögert. Dagegen ist bei der Seruminsulinkonzentration eine deutliche Beziehung zwischen Glucosedosis und Insulinausschüttung festzustellen. Nach 50 g Glucose beträgt der durchschnittliche maximale Anstieg des Seruminsulins etwa $60 \mu \mathrm{E} / \mathrm{ml}$, nach $100 \mathrm{~g}$ Glucose etwa $100 \mu \mathrm{E} / \mathrm{ml}$, nach $200 \mathrm{~g}$ Glucose etwa $120 \mu \mathrm{E} / \mathrm{ml}$. Die Normalisierung der Seruminsulinkonzentration erfolgt bei den größeren Glucosemengen deutlich verzögert. $120 \mathrm{~min}$ nach Einnahme von $200 \mathrm{~g}$ Glucose ist die Seruminsulinkonzentration höher als die maximale Seruminsulinkonzentration nach $50 \mathrm{~g}$ Glucose. Trotzdem sind nach $120 \mathrm{~min}$ die Blutglucosewerte auch nach der hohen Dosierung wieder annähernd normalisiert. Auch nach $100 \mathrm{~g}$ Glucose ist das Seruminsulin 2 Std nach Einnahme der Glucose bei normalen Blutglucosewerten noch auf das Vierfache des Nüchternwertes erhöht.

Nach oraler Verabreichung eines Stärkehydrolysates, das je zur Hälfte aus Maltose und aus höheren Polysacchariden bestand (siehe Tabelle 1), wurden

Tabelle 1

\begin{tabular}{lc}
\hline Stärkehydrolysat, Glucosesirup & Mor Sweet 1424 \\
\hline Trockensubstanz & $77.5 \%$ \\
Dextrose-Äquivalent & 40 \\
Kohlenhydratzusammensetzung der Trockensubstanz \\
Dextrose & $6 \%$ \\
Maltose & $54 \%$ \\
höhere molekulare Zucker & $40 \%$
\end{tabular}
(nach Angabe der Maizena-Werke, durch eigene papierchromatographische Analyse bestätigt)

praktisch identische Ergebnisse erzielt (Abb. 2). Die Blutglucosekonzentrationen zeigten keine wesentlichen Unterschiede gegenüber den Belastungen mit Glucose. Auch die Seruminsulinwerte sind nur teilweise geringfügig niedriger. Die gesamte Insulinausschüttung, die als Integral des Seruminsulinanstieges bestimmt werden kann [13], zeigt eine lineare Beziehung zur verabreichten Glucosemenge. Die Beziehung zwischen Blutglucosekonzentration und Seruminsulinkonzentration ist statistisch nicht sehr deutlich. Hier mag auch die relativ große Fehlerbreite und die geringe Stichprobenanzahl der Seruminsulinbestimmung von Bedeutung sein.

An statistischen Parametern wurde der Regressionskoeffizient b (Blutglucosekonzentration als unabhängige Variable, Seruminsulinkonzentration als abhängige Variable) mit zugehöriger Konfidenz $l$ sowie der Korrelationskoeffizient $r$ mit Konfidenz- 

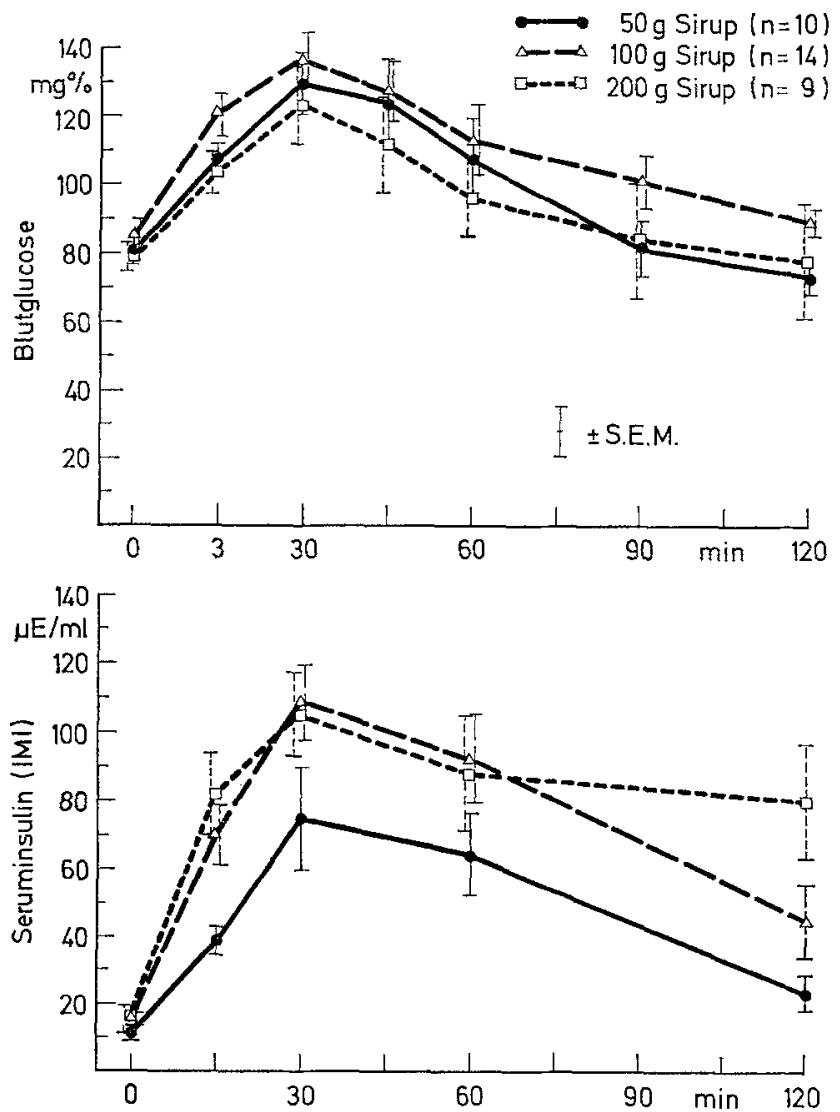

Abb. 2. Verhalten der Blutglucose und des Seruminsulins (IMI) bei jungen gesunden Versuchspersonen nach oraler Verabreichung von Glucosesirup verworfen werden. Daraus folgt, daß einem bestimmten Blutglucosewert kein entsprechender Seruminsulinwert zugeordnet werden kann. Prüft man die Parameter unter Vernachlässigung der Zeit, so erhält man zwar einen ausreichend großen Korrelationskoeffizient; hierbei ist aber nicht auszuschließen, daß durch die Zusammennahme der verschiedenen Gruppen eine Inhomogenität wirksam wird. Der Regressionskoeffizient und auch der Korrelationskoeffizient sind am größten bei den 60 min-Werten. Hier ist also die am meisten gesicherte Beziehung zwischen den beiden verglichenen Werten vorhanden.

Wenn man die Nüchternwerte mit den Werten zum entsprechenden Zeitpunkt in eine Gruppe nimmt, erhält man eine bessere Korrelation (siehe Tabelle 3). In diesem Fall werden letztlich Blutglucoseanstieg und Seruminsulinanstieg verglichen. Da es sich um den Vergleich von zwei Gruppen von Meßwerten handelt, wird hier eine Inhomogenität in Kauf genommen. Dabei ist die Beziehung bei den 30 min-Werten am besten. Es kann gefolgert werden, daß die Insulinsekretion nach 15 min noch nicht in einem so starken Ausmaß auf die Veränderung der Blutglucosekonzentration reagiert hat wie nach $30 \mathrm{~min}$. Zusätzlich scheint die gesteigerte periphere Glucoseaufnahme als Folge der Insulinsekretion auch erst nach diesem Zeitpunkt die Korrelation zwischen Blutglucose und Seruminsulin zu verschlechtern.

\section{Diskussion}

Bei oralen Kohlenhydratbelastungen ist die intestinale Glucoseresorption die Störgröße im

Tabelle 2. a) Beziehung zwischen Blutglucose und Seruminsulin (IMI) nach oralen Belastungen mit Glucose $(50 \mathrm{~g}, 100 \mathrm{~g}, 200 \mathrm{~g})$, bzw. mit Stärkehydrolysat $(50 \mathrm{~g}, 100 \mathrm{~g}$, $200 \mathrm{~g}$ ) ohne Berïcksichtigung der Dosierung. Berechnung für $p<0.05$

\begin{tabular}{|c|c|c|c|c|c|c|}
\hline & Zeit & $r$ & Konfide & tervall & $b$ & $l$ \\
\hline Glucose & $\begin{array}{r}0 \\
15 \mathrm{~min} \\
30 \mathrm{~min} \\
60 \mathrm{~min} \\
120 \mathrm{~min}\end{array}$ & $\begin{array}{r}-0.07 \\
0.22 \\
0.34 \\
0.48 \\
0.31\end{array}$ & $\begin{array}{r}-0.41 \\
-0.14 \\
-0.01 \\
0.16 \\
-0.05\end{array}$ & $\begin{array}{l}0.29 \\
0.53 \\
0.62 \\
0.71 \\
0.60\end{array}$ & $\begin{array}{r}-0.02 \\
0.26 \\
0.41 \\
0.67 \\
0.51\end{array}$ & $\begin{array}{l}0.11 \\
0.37 \\
0.36 \\
0.38 \\
0.50\end{array}$ \\
\hline $\begin{array}{l}\text { obne } \\
\text { der } 2\end{array}$ & rücksichtigung & 0.55 & 0.43 & 0.65 & 0.72 & 0.15 \\
\hline $\begin{array}{l}\text { Stärke- } \\
\text { hydrolysat }\end{array}$ & $\begin{array}{l}0 \\
15 \mathrm{~min} \\
30 \mathrm{~min} \\
60 \mathrm{~min} \\
120 \mathrm{~min}\end{array}$ & $\begin{array}{l}0.24 \\
0.12 \\
0.21 \\
0.23 \\
0.11\end{array}$ & $\begin{array}{r}-0.14 \\
-0.26 \\
-0.18 \\
-0.17 \\
-0.28\end{array}$ & $\begin{array}{l}0.55 \\
0.46 \\
0.54 \\
0.56 \\
0.47\end{array}$ & $\begin{array}{l}0.08 \\
0.17 \\
0.26 \\
0.29 \\
0.20\end{array}$ & $\begin{array}{l}0.11 \\
0.47 \\
0.42 \\
0.42 \\
0.59\end{array}$ \\
\hline $\begin{array}{l}\text { ohne } \\
\text { der }\end{array}$ & ücksichtigung & 0.42 & 0.28 & 0.55 & 0.62 & 0.19 \\
\hline
\end{tabular}

intervall bestimmt [14]. Bei der direkten Auswertung der Größen (siehe Tabelle 2) wurde ein sehr niedriger Korrelationskoeffizient mit sehr großem Konfidenzintervall festgestellt. Bei der kleinen Zahl der Beobachtungen kann also die Hypothese, es bestehe kein Zusammenhang zwischen den beiden Größen, nicht
Regellkreis der Blutzuckerregulation. Es ist vorauszusehen, daß die Stellgröße Seruminsulinkonzentration gegenüber der Regelgröße Blutglucosekonzentration verzögert anspricht. Dies geht auch aus früheren Untersuchungen, z.B. von Luft [9], hervor. Andererseits ist überraschend, daß die Regelgröße Blut- 
glucosekonzentration bereits annähernd normalisiert ist, obwohl die Stellgröße noch auf das Achtfache der Norm erhöht sein kann. Daraus ist zu schließen, daß nicht nur die Blutglucose als solche, sondern auch andere Faktoren auf die Insulinsekretion einwirken. Auf die höhere Insulinausschüttung nach oraler Glucoseverabreichung gegenüber intravenöser Ver- gänge zu erwarten. Der Regelkreis ist offensichtlich darauf eingerichtet, die Blutglucosekonzentration auf ein bestimmtes Niveau einzuregulieren. Da die Stellgröße Seruminsulin jedoch verzögert auf die Veränderung der Regelgröße Blutglucose reagiert, kommt es initial zu einer überschießenden Erhöhung der Blutglucosekonzentration. Anschließend wird trotz

b) Beziehung zwischen Blutglucose und Seruminsulin (IMI) nach oralen Belastungen mit Glucose unter Berücksichtigung der Dosierung. Berechnung für $p<0.05$

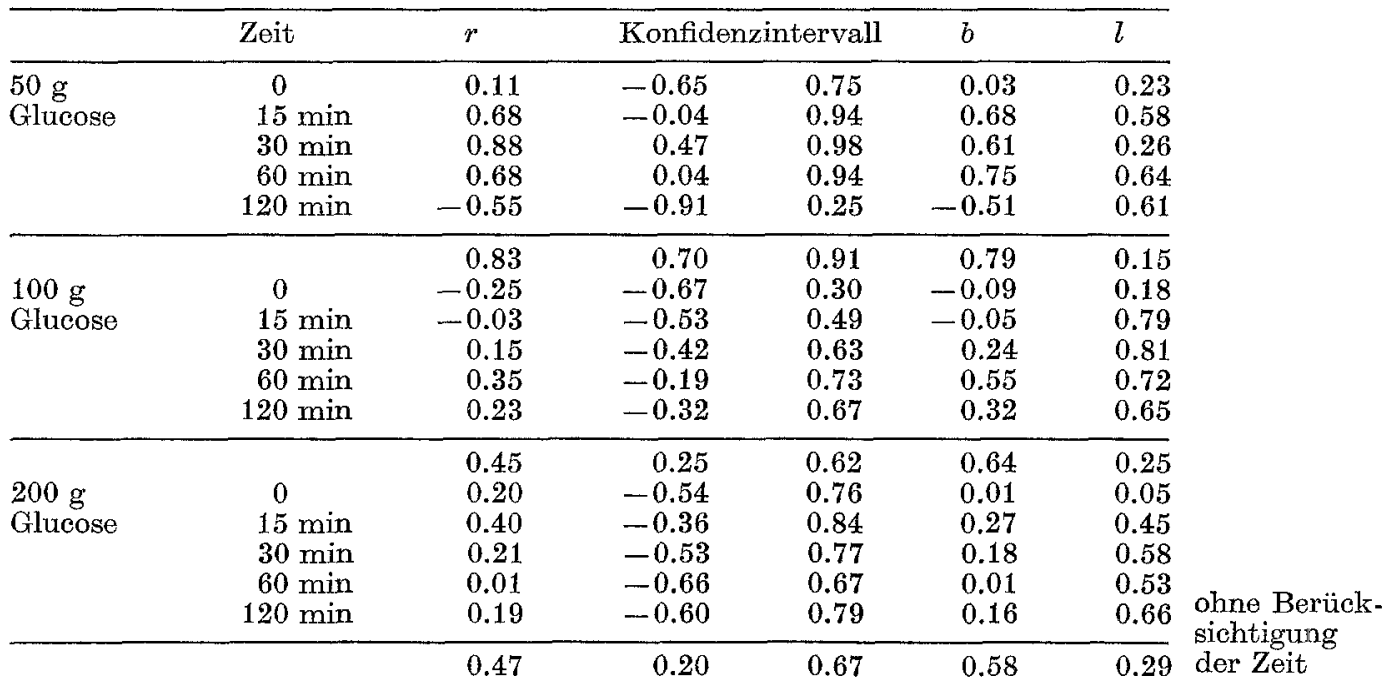

c) Beziehung zwischen Blutglucose und Seruminsulin (IMI) nach oralen Belastungen mit Stärkehydrolysat unter Berücksichtigung der Dosierung. Berechnung für $p<0.05$

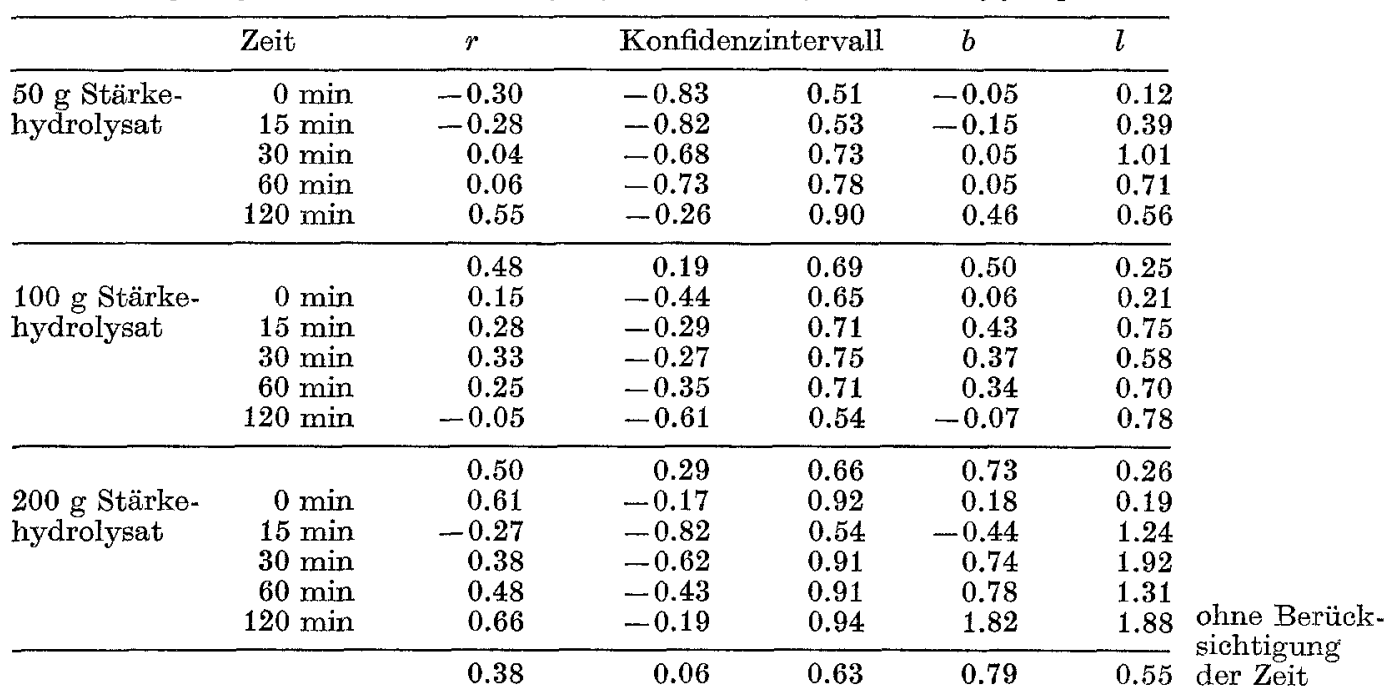

abreichung wurde schon früher von verschiedenen Autoren, z.B. von Ellrick und Mitarb. [2], sowie Seltzer und Mitarb. [15] hingewiesen. Bei den oralen Glucosebelastungen scheint den gastrointestinalen Faktoren [3, 12] eine wesentliche Rolle bei der Stimulierung der Insulinsekretion zuzukommen. Unter physiologischen Bedingungen ist eine Störung der Glucosehomoiostase nur durch die Resorptionsvor- offensichtlich andauernder Glucoseresorption die Blutglucosekonzentration wieder normalisiert. Dieser Rückschluß ist auch aus Ergebnissen von oralen Galaktosebelastungen möglich. Galaktose wird wahrscheinlich über den gleichen Mechanismus und genauso schnell wie Glucose resorbiert [1]. Da die Galaktose insulinunabhängig verwertet wird, ist ein Ansteigen der Galaktosekonzentration im Blut nach 
oraler Galaktoseapplikation beweisend für die Galaktoseresorption [5]. Wie wir früher zeigen konnten, ist die Galaktoseresorption nach Verabreichung von $200 \mathrm{~g}$ Galaktose nach $240 \mathrm{~min}$ sicher noch nicht abgeschlossen [5]. Für die Glucoseresorption sind ähnliche Ver- die Magenentleerung limitierend [10]. Es wäre vorstellbar, daß die fast identischen Ergebnisse unserer Untersuchungen nach oralen Belastungen mit Glucose bzw. mit Stärkehydrolysat auf eine veränderte Ma. genentleerung zurückzuführen sind.

Tabelle 3. a) Beziehung zwischen Blutglucose und Seruminsulin (IMI) nach oralen Belastungen mit Glucose $(50 \mathrm{~g}, 100 \mathrm{~g}, 200 \mathrm{~g})$, bzw. mit Stärkehydrolysat $(50 \mathrm{~g}, 100 \mathrm{~g}$, $200 \mathrm{~g}$ ) ohne Berücksichtigung der Dosierung. Vergleich mit Ausgangswert. Berechnung für $p<0.05$

\begin{tabular}{|c|c|c|c|c|c|c|}
\hline Zeit & $r$ & \multicolumn{2}{|c|}{ Konfidenzintervall } & $b$ & $l$ & \\
\hline $0 / 15$ min & 0.66 & 0.49 & 0.78 & 0.81 & 0.20 & \\
\hline $0 / 30 \mathrm{~min}$ & 0.72 & 0.58 & 0.82 & 0.93 & 0.19 & \\
\hline $0 / 60 \mathrm{~min}$ & 0.57 & 0.37 & 0.71 & 0.97 & 0.29 & Belastung mit \\
\hline $0 / 120 \mathrm{~min}$ & 0.24 & -0.01 & 0.46 & 0.41 & 0.34 & Glucose \\
\hline $0 / 15 \mathrm{~min}$ & 0.53 & 0.32 & 0.69 & 0.77 & 0.27 & \\
\hline $0 / 30 \mathrm{~min}$ & 0.66 & 0.48 & 0.79 & 0.99 & 0.25 & Belastung mit \\
\hline $0 / 60 \mathrm{~min}$ & 0.45 & 0.22 & 0.64 & 0.75 & 0.33 & Stärke- \\
\hline $0 / 120 \min$ & 0.13 & -0.14 & 0.38 & 0.24 & 0.40 & hydrolysat \\
\hline
\end{tabular}

b) Beziehung zwischen Blutglucose und Seruminsulin (IMI) nach oralen Belastungen mit Glucose unter Berücksichtigung der Dosierung. Vergleich mit Ausgangswert. Berechnung für $p<0.05$

\begin{tabular}{llccccc}
\hline & Zeit & $r$ & \multicolumn{2}{c}{ Konfidenzintervall } & $b$ & $l$ \\
\hline $50 \mathrm{~g}$ & $0 / 15 \mathrm{~min}$ & 0.84 & 0.59 & 0.94 & 0.91 & 0.28 \\
Glucose & $0 / 30 \mathrm{~min}$ & 0.90 & 0.73 & $\mathbf{0 . 9 7}$ & 0.81 & 0.18 \\
& $0 / 60 \mathrm{~min}$ & 0.64 & 0.21 & 0.86 & 0.72 & 0.41 \\
& $0 / 120 \mathrm{~min}$ & -0.33 & -0.71 & $\mathbf{0 . 2 0}$ & -0.22 & 0.30 \\
\hline $100 \mathrm{~g}$ & $0 / 15 \mathrm{~min}$ & 0.51 & 0.18 & 0.74 & 0.72 & 0.39 \\
Glucose & $0 / 30 \mathrm{~min}$ & 0.63 & 0.35 & 0.81 & 0.91 & 0.36 \\
& $0 / 60 \mathrm{~min}$ & 0.44 & 0.10 & 0.69 & 0.86 & 0.56 \\
& $0 / 120 \mathrm{~min}$ & 0.15 & -0.22 & $\mathbf{0 . 4 8}$ & 0.21 & 0.44 \\
\hline $200 \mathrm{~g}$ & $0 / 15 \mathrm{~min}$ & 0.77 & 0.48 & 0.91 & 0.86 & 0.31 \\
Glucose & $0 / 30 \mathrm{~min}$ & 0.77 & 0.47 & 0.91 & 1.05 & $\mathbf{0 . 3 8}$ \\
& $0 / 60 \mathrm{~min}$ & $\mathbf{0 . 5 8}$ & $\mathbf{0 . 1 5}$ & 0.82 & 0.95 & $\mathbf{0 . 5 9}$ \\
& $0 / 120 \mathrm{~min}$ & 0.29 & -0.23 & 0.67 & 0.59 & 0.89 \\
\hline
\end{tabular}

c) Beziehung zwischen Blutglucose und Seruminsulin (IMI) nach oralen Belastungen mit Stärkehydrolysat unter Berücksichtigung der Dosierung. Vergleich mit Ausgangswert. Berechnung für $p<0.05$

\begin{tabular}{lllllll}
\hline & Zeit & $r$ & \multicolumn{2}{l}{ Konfidenzintervall } & $b$ & $l$ \\
\hline 50 g Stärke- & o/ $15 \mathrm{~min}$ & 0.41 & -0.11 & $\mathbf{0 . 7 5}$ & 0.32 & $\mathbf{0 . 3 3}$ \\
hydrolysat & $0 / 30 \mathrm{~min}$ & 0.58 & 0.11 & $\mathbf{0 . 8 3}$ & 0.71 & 0.47 \\
& $0 / 60 \mathrm{~min}$ & 0.43 & -0.11 & $\mathbf{0 . 7 7}$ & 0.47 & 0.49 \\
& $0 / 120 \mathrm{~min}$ & 0.24 & -0.29 & $\mathbf{0 . 6 6}$ & 0.19 & 0.36 \\
\hline 100 g Stärke- & $0 / 15 \mathrm{~min}$ & 0.65 & $\mathbf{0 . 3 6}$ & $\mathbf{0 . 8 3}$ & 0.93 & 0.37 \\
hydrolysat & $0 / 30 \mathrm{~min}$ & 0.74 & 0.49 & $\mathbf{0 . 8 7}$ & 1.06 & 0.34 \\
& $0 / 60 \mathrm{~min}$ & 0.53 & 0.17 & $\mathbf{0 . 7 6}$ & 0.90 & 0.51 \\
& $0 / 120 \mathrm{~min}$ & 0.13 & -0.28 & $\mathbf{0 . 5 0}$ & 0.18 & 0.49 \\
\hline 200 g Stärke- & $0 / 15 \mathrm{~min}$ & $\mathbf{0 . 4 3}$ & -0.09 & $\mathbf{0 . 7 6}$ & 0.81 & 0.81 \\
hydrolysat & $0 / 30 \mathrm{~min}$ & 0.72 & 0.30 & $\mathbf{0 . 9 0}$ & $\mathbf{1 . 7 7}$ & $\mathbf{0 . 8 9}$ \\
& $0 / 60 \mathrm{~min}$ & $\mathbf{0 . 4 2}$ & -0.12 & $\mathbf{0 . 7 7}$ & 0.95 & $\mathbf{1 . 0 1}$ \\
& $0 / 120 \mathrm{~min}$ & $\mathbf{0 . 2 3}$ & -0.32 & $\mathbf{0 . 6 6}$ & 0.72 & $\mathbf{1 . 5 3}$ \\
\hline
\end{tabular}

hältnisse zu erwarten. Die intestinale Resorption von Maltose und Stärkehydrolysaten erfolgt nach eigenen Untersuchungen wesentlich langsamer als die Glucoseresorption (Förster, unveröffentlicht). Allerdings ist für die Resorption rasch resorbierbarer Substanzen
Nach unseren Ergebnissen ist die orale Glucosebelastung mit $100 \mathrm{~g}$ Glucose zu diagnostischen Zwecken der oralen Belastung mit nur $50 \mathrm{~g}$ Glucose überlegen, da sie zu einer stärkeren und vor allem länger anhaltenden Stimulierung der Insulinsekretion fïhrt. Bei 
noch höheren Glucosedosierungen wird bei gesunden Versuchspersonen keine weitere Erhöhung der maximalen Seruminsulinkonzentration mehr bewirkt. Den Glucosekonzentrationen nach $15 \mathrm{~min}$ und $30 \mathrm{~min}$ kommt keine wesentliche diagnostische Bedeutung $\mathrm{zu}$, da hier der Einfluß der Störgröße noch zu groß ist. Nach 120 min ist dagegen bei gesunden Versuchspersonen nach oraler Verabreichung von $50 \mathrm{~g}$ Glucose die Stellgröße bereits normalisiert, $d . h$. die Blutglucosekonzentration zu diesem Zeitpunkt hat bei dieser Konzentration keine wesentliche Aussagekraft. Die Glucoseresorption ist offensichtlich abgeschlossen, eine Provokation der Insulinsekretion liegt nicht mehr vor.

Zur Feststellung einer eingeschränkten GlucoseInsulin-Homoiostase - also in der Diabetesdiagnostik - ist daher die höhere Glucosedosierung vorteilhaft. Es ist zu erwarten, daß nach Verabreichung von $100 \mathrm{~g}$ Glucose der allgemein als besonders wichtig anerkannte Zweistunden-Blutglucosewert bei einer Störung des Kohlenhydratstoffwechsels eher pathologisch erhöht ist, als nach Verabreichung von $\mathbf{5 0} \mathrm{g}$ Glucose.

Die Verwendung des geschmacklich vorteilhafteren und womöglich besser bekömmlichen Stärkehydrolysats für orale Belastungen ist nach diesen Untersuchungen gerechtfertigt, wenn auch nicht erforderlich. In der Diabetesdiät hat das verwendete Stärkehydrolysat die gleichen Nachteile hinsichtlich der Beeinflussung des Kohlenhydratstoffwechsels wie Glucose.

\section{Anmerkung bei der Korreltur:}

In einer weiteren Untersuchungsreihe wurde zusätzlich zu den hier angeführten Parametern noch die Konzentration der Freien Fettsäuren (FFS) und die Glucosekonzentration im Kapillarblut bestimmt.

Aus den Ergebnissen kann gefolgert werden, daß 2 Std nach oraler Verabreichung von $30 \mathrm{~g}$ oder $50 \mathrm{~g}$ Glucose keine wesentliche periphere Insulinwirkung mehr nachzuweisen ist (geringe kapillar-venöse Glucosekonzentrationsdifferenzen, Ansteigen der Konzentration der FFS). 2 Std nach Verabreichung von 100 g Glucose (oder mehr) konnte dagegen bei signifikant erhöhter Seruminsulin. konzentration eine große kapillar-venöse Glucosekonzentrationsdifferenz und eine noch andauernde Erniedrigung der Konzentration der FFS als Ausdruck einer peripheren Insulinwirkung festgestellt werden. Es ist anzunehmen, daß der nachweisbare periphere Glucoseabstrom bei fast normalisierter Blutglucosekonzentration durch einen entsprechenden Glucoseeinstrom aus dem Intestinalbereich kompensiert wird.

(Förster, H., M. Haslbeck, H. Mehnert: Vortrag 7. Congress International Diabetes Federation, Buenos Aires 23. -28 . 8. 1970).

\section{Literatur}

1. Crane, R.K.: Intestinal absorption of sugars. Physiol. Rev. 40, 789-824 (1960).

2. Elrick, H., Stimmler, L., Hlad, C.J., Arai, Y.: Plasma insulin response to oral and intravenous glucose administration. J. clin. Endocr. 24, 1076-1083 (1964).

3. Fasel, J., Hadjikani, H. : Le role endocrinien du duodenum et de l'intestin grele. Schweiz. med. Wschr. 98, 133-141 (1969).

4. Förster, H., Mehnert, H.: Orale und intraduodenale Glucose - sowie Fructosebelastung mit verschiedener Dosierung. Verh. d. Ges. inn. Med. 73. Kongress 1967, S. 778.

5. - Haslbeck, M., Mehnert, H.: Untersuchungen an Gesunden und an leberkranken Patienten nach intravenöser und oraler Galaktosebelastung. Verh. dtsch. Ges. inn. Med. 73. Kongress 1967, S. 245.

6. Goetz, F.C., Maney, J.W., Greenberg, B.Z.: The regulation of insulin secretion: effects of the infusion of glucose, ribose and other sugars into the portal veins of dogs. J. Lab. clin. Med. 69, 537- 543 (1967)

7. Hales, C.N. Randle, P.J.: Immunoassay of insulin with insulinantibody precipitate. Biochem. J. 88, 137 (1963)

8. Huggett, S., Nixon, D.A. : Enzymic determination of blood glucose. Biochem. J. 66, 12 P-13 P (1957).

9. Luft, R.: "What is inherited, what is added" Hypothesis for the pathogenesis of diabetes mellitus. Diabetes 16, 615-619 (1967).

10. Mehnert, H., Förster, H.: Untersuchungen über den Mechanismus der Magenentleerung bei Mensch und Ratte nach oraler Applikation verschiedener .Zucker. Diabetologia 4, 26-33 (1968).

11. Perley, M., Kipnis, D.M. : Plasma insulin responses to glucose and tolbutamide of normal weight and obese diabetic and nondiabetic subjects. Diabetes 15, 867875 (1966).

12. Pfeiffer, E.F., Raptis, S.: Intestinale Hormone und Insulinsekretion. Klin. Wschr. 46, 337-343 (1968).

13. Reaven, G., Miller, R.: Study of the relationship between glucose and insulin responses to an oral glucose load in man. Diabetes 17, 560-566 (1968).

14. Sachs, L.: Statistische Auswertungsmethoden. Berlin, Heidelberg, New York, Springer 1968.

15. Seltzer, H.S., Allen, E.W., Herron, A.L., Brennan, M.T.: Insulin secretion in response to glycemic stimulus : relation of delayed initial release to carbohydrate intolerance in mild diabetes mellitus. J. clin. Invest. 46, 323-330 (1967).

H. Förster

Institut für vegetative

Physiologie der Universität

D.6 Frankfurt

Ludwig-Rehn-Str. 14 